\title{
Designing Smart Environments: A Paradigm Based on Learning and Prediction
}

\author{
Sajal K. Das and Diane J. Cook \\ Department of Computer Science and Engineering, \\ The University of Texas at Arlington \\ \{das, cook\}@cse.uta.edu
}

\begin{abstract}
We propose a learning and prediction based paradigm for designing smart home environments. The foundation of this paradigm lies in information theory as it manages uncertainties of the inhabitants' contexts (e.g., locations or activities) in daily lives. The idea is to build compressed dictionaries of contextaware data collected from sensors and devices monitoring and/or controlling the smart environment, efficiently learn from these profiles, and finally predict inhabitant's future contexts. Successful prediction helps automate device control operations and tasks within the environment as well as to identify anomalies. Thus, the learning and prediction based paradigm optimizes such goal functions of smart environments as minimizing maintenance cost, manual interactions and energy utilization. After identifying important features of smart environments, we present an overview of our MavHome architecture and apply the proposed paradigm to the inhabitant's location and activity tracking and prediction, and automated decision-making capability.
\end{abstract}

\section{Introduction}

We live in an increasingly connected and automated society. Smart environments embody this trend by linking computers and other devices to everyday settings and commonplace tasks. Although the desire to create smart environments has existed for decades, research on this multidisciplinary topic has become increasingly intense in the recent years. Indeed, tremendous advances in smart devices, wireless mobile communications, sensor networks, pervasive computing, machine learning, robotics, middleware and agent technologies, and human computer interfaces have made the dream of smart environments a reality. To our understanding, a smart environment is a small world where sensor-enabled and networked devices work continuously and collaboratively to make lives of inhabitants more comfortable. "Smart" or "intelligent" means "the ability to autonomously acquire and apply knowledge", while an "environment" refers to our surroundings. Thus, a "smart environment" is able to acquire and apply knowledge about an environment and adapt to its inhabitants, thereby improving their experience [7].

The type of experience that individuals wish from an environment varies with the individual and the type of environment considered. This may include the safety of inhabitants, reduction of cost of maintaining the environment, optimization of resources (e.g., utility/energy bills or communication bandwidth), or task automation. 
Reflecting the increased interest in smart environments, research labs in academia and industry are picking up the theme and creating environments with their own individual spin and market appeal. For example, the Aware Home [1, 21], Adaptive House [26], and MavHome [9, 32] use sensors to learn models of the inhabitants and automate activities accordingly. Other designs include smart offices, classrooms, kindergartens, tables, and cars $[1,3,13,22,30]$. Connected homes with device communications capability have become the focus of companies such as Philips, Cisco [5], Verizon, Sun, Ericsson, and Microsoft [4]. Projects on smart environments to assist individuals with health challenges are discussed in [10, 12, 14, 17, 20]. Refer to [7], for a comprehensive treatment of necessary technologies, architectures, algorithms, and protocols to build smart environments for a variety of applications.

This paper presents our research experience in developing MavHome [9, 32], a smart home project funded by the US National Science Foundation. In particular, we propose "learning and prediction" as a paradigm for designing efficient algorithms and smart protocols in smart environments. This paradigm lies in information theory as it manages inhabitants' uncertainties in mobility and activities in daily lives. The underlying idea is to build intelligent (compressed) dictionaries of inhabitants' mobility and activity profiles collected from sensor data, learn from this information, and predict future mobility and actions. Such prediction helps device automation and efficient resource management, thus optimizing the goals of the smart environment.

\section{Features of Smart Environments}

Important features of smart environments are that they possess a degree of autonomy, adapt themselves to changing environments, and communicate with humans in a natural way [7]. Intelligent automation can reduce the amount of interactions required by the inhabitants, as well as reduce resource consumption and other potential wastages. These capabilities can provide additional features such as detection of unusual or anomalous behavior for health monitoring and home security, for example.

Remote Control of Devices: The most basic feature is the ability to control devices remotely or automatically. By plugging devices into simple power-line controllers like X10, inhabitants can turn lights, coffee makers, and other appliances on or off in much the same way as couch potatoes switch television stations with a remote control. Computer software can additionally be employed to program sequences of device activities and capture device events. This capability allows inhabitants to be free from the requirement of physical access to devices. Individuals with disabilities can control devices from a distance. Automated lighting sequences can give the impression that an environment is occupied while inhabitants are gone, thus handling routine procedures without human intervention.

Device Communications: With the maturity of wireless communications and middleware technology, smart environment designers and inhabitants have been able to raise their standards and expectations. In particular, devices use these technologies to communicate with each other, share data to build a more informed model of the state of the environment and/or inhabitants, and retrieve information from outside sources over the Internet or wireless network infrastructure. With these capabilities, 
for example, the environment can access the weather page to determine the forecast and query the moisture sensor in the lawn to determine how long the sprinklers should run. Devices can access information from the Internet such as menus, operational manuals, or software upgrades, and can post information such as a grocery store list generated from monitoring inventory with an intelligent refrigerator or trash bin.

Activation of one device can also trigger other sequences, such as turning on the bedroom radio, kitchen coffee maker, and bathroom towel warmer when the alarm goes off. Inhabitants can benefit from the interaction between devices by muting the television sound when the telephone or doorbell rings; temperature as well as motion sensors can interact with other devices to ensure that the temperature is kept at a desired level wherever the inhabitants are located within the environment.

Sensory Information Acquisition: Recent advancements in sensor technology have made it possible to make low-level decisions from monitored data. As a result, environments can provide dynamic adjustments based on sensor readings and can better customize behaviors to the nuances of the inhabitants' surroundings. Motion detectors or force sensors can detect the presence of individuals in the environment and accordingly adjust lights, music, or climate control. Water and gas sensors can monitor potential leaks and force the valves, thus closing them when a danger arises. Low-level control of devices offers fine-tuning in response to changing conditions, such as adjusting window blinds as the amount of daylight coming into a room changes. Networks composed of these sensors can share data and offer information to the environment at speeds and complexity not experienced before. For example, a Smart Sofa [29] can identify individuals based on the weight and thus customize device settings around the house.

Enhanced Services by Intelligent Devices: Smart environments are usually equipped with numerous networked and sensor-enabled devices/appliances that provide varied and impressive capabilities. For example, Frigidaire and Whirlpool offer intelligent refrigerators with features that include web cameras to monitor inventory, bar code scanners, and Internet-ready interactive screens. Through interactive cameras, inhabitants away from home can view the location of security or fire alerts; similarly remote caregivers can check on the status of their patients or family. Merloni's washing machine uses sensor information to determine appropriate cycle times. In addition, specialized equipments have been designed in response to the growing interest in assistive environments. Researchers at MIT's Media Lab are investigating new specialized devices, such as an oven mitt that can tell if food has been warmed all the way through. A breakthrough development from companies such as Philips is an interactive tablecloth that provides cable-free power to all chargeable objects placed on the table's surface. An environment that can combine the features of these devices with information gathering and remote control capability will realize many of the intended goals of smart environment designers.

Predictive Decision Making Capabilities: Full automation and adaptation of smart environments rely on the software itself to learn, or acquire information that allows the software to improve its performance with experience. Specific features of recent smart environments that meet these criteria incorporate predictive and automatic decision-making capabilities into the control paradigm. Contexts (mobility or activity) of inhabitants as well as of the environment can be predicted with good accuracy based 
on observed activities and known features. Models can also be built of inhabitant patterns that can be used to customize the environment for future interactions. For example, an intelligent car can collect information about the driver including typical times and routes to go to work, theatre, restaurant, and store preferences, and commonly used gas stations. Combining this information with data collected by the inhabitant's home and office as well as Internet-gathered specifics on movie times, restaurant menus and locations, and sales at various stores, the car can make recommendations based on the learned model of activity patterns and preferences. Similarly, building device performance model can allow the environment to optimize its behaviors and performance. For example, smart light bulbs may warn expiry time, letting the factory deliver replacements before the need is critical.

As a complement to predictive capabilities, a smart environment will be able to decide on how to automate its own behaviors to meet the specified goals. The environment should control device settings and timings; it should also elect between alternate methods of achieving a goal, such as turning on lights in each room entered by an inhabitant or anticipating where the inhabitant is heading and illuminating just enough of the environment to direct the individual to their goal.

\section{The MavHome Smart Home}

The MavHome $[9,32]$ at the University of Texas at Arlington represents an environment that acts as an intelligent agent, perceiving the state of the home through sensors and acting upon the environment through device controllers with a goal to maximize inhabitants' comfort and minimize home's operating cost. To achieve this goal, the house must reason about, learn, predict, and adapt to its inhabitants.

In MavHome, the desired smart home capabilities are organized into an agent based software architecture that seamlessly connects the components. Figure 1 describes the architecture of a MavHome agent that separates the technologies and functions into four cooperating layers: (i) the Decision layer selects actions for the

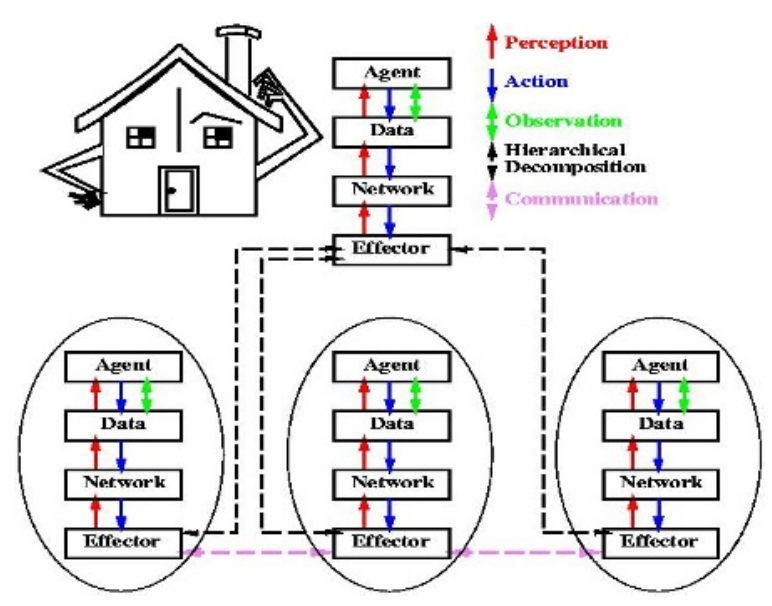

Fig. 1. MavHome agent architecture 
agent to execute; (ii) the Information layer collects information and generates inferences useful for making decisions; (iii) the Communication layer is responsible for routing and sharing information between agents; and (iv) the Physical layer consists of devices, transducers, and network equipments. The MavHome software components are connected using a distributed inter-process communication interface. Because controlling an entire house is a large-scale complex learning and reasoning problem, it is decomposed into reconfigurable tasks. Thus, the Physical layer for one agent may represent another agent somewhere in the hierarchy, which is capable of executing the task selected by the requesting agent.

Perception is a bottom-up process. Sensors monitor the environment (e.g., lawn moisture level) and transmit information to another agent through the Communication layer. The database records the information in the Information layer, updates its learned concepts and predictions, and alerts the Decision layer of the presence of new data. During action execution, information flows top down. The Decision layer selects an action (e.g., run the sprinklers) and relates the decision to the Information layer. After updating the database, the Communication layer routes the action to the appropriate effector to execute. Specialized interface agents allow interaction with users and external resources such as the Internet. Agents communicate with each other using the hierarchical flow as shown in Fig. 1. In the following, a smart home will generically represent a smart environment.

\section{Automation Through Learning and Prediction}

In order to maximize comfort, minimize cost, and adapt to the inhabitants, a smart home must rely upon sophisticated tools for intelligence building such as learning, prediction, and making automated decisions. We will demonstrate that learning and prediction indeed play an important role in determining the inhabitant's next action and anticipating mobility patterns within the home. The home will need to make this prediction based solely on the history of mobility patterns and previously seen inhabitant interactions with various devices (e.g., motion detectors, sensors, device controllers, video monitors), as well as the current state of the inhabitant and/or the house. The captured information can be used to build powerful models that aid in efficient prediction algorithms. The number of prediction errors must be minimal, and the algorithms must be able to deliver predictions with minimal processing delays. Prediction is then handed over to a decision-making algorithm that selects actions for the house to meet its desired goals. The underlying concepts of MavHome prediction schemes lie in the text compression, on-line parsing and information theory. Wellinvestigated text compression methods $[8,31]$ have established that good compression algorithms are also good learners and hence good predictors. According to information theory [8], a predictor with an order (size of history used) that grows at a rate approximating the entropy rate of the source is an optimal predictor. We summarize below a novel paradigm for inhabitant's mobility and activity predictions.

\subsection{Inhabitant Location Prediction}

Location is perhaps the most common example of context. Hence, it is crucial for a smart environment to track inhabitant's mobility accurately by determining and 
predicting his location. The prediction also helps in optimal allocation of resources and activation of effectors in location-aware applications [11, 24]. In [2], we proposed a model-independent algorithm for location prediction in wireless cellular networks, which we later adopted for indoor location tracking and predicting inhabitant's future locations [15, 28]. Our approach uses symbolic representation of location information that is relative to the access infrastructure topology (e.g., sensor ids or zones through which the inhabitant passes), making the approach universal or model-independent. At a conceptual level, prediction involves some form of statistical inference, where some sample of the inhabitant's movement history (profile) is used to provide intelligent estimates of future location, thereby reducing the location uncertainty associated with the prediction $[11,27]$.

Hypothesizing that the inhabitant's mobility has repetitive patterns that can be learned, and assuming the inhabitant's mobility process as stochastically random, we proved that [2]: It is impossible to optimally track mobility with less information exchange between the system (i.e., smart environment) and the device (detecting inhabitant's mobility) than the entropy rate of the stochastic mobility process. Specifically, given the past observations of inhabitant's position and the best possible predictors of future position, some uncertainty in the position will always exist unless the device and the system exchange location information. The actual method by which this exchange takes place is irrelevant to this bound. All that matters is that the exchange exceeds the entropy rate of the mobility process. Therefore, a key issue in establishing bounds is to characterize the mobility process (and hence entropy rate) in an adaptive manner. To this end, based on information-theoretic framework, we proposed an optimal on-line adaptive location management algorithm, called LeZi-update [2]. Rather than assuming a finite mobility model, LeZi-update learns his movement history stored in a Lempel-Ziv type of compressed dictionary [31], builds a universal model by minimizing entropy, and predicts future locations with high accuracy. In other words, LeZi-update offers a model-independent solution to manage mobility related uncertainty. This framework is also applicable to other contexts such as activity prediction [16], resource provisioning [11, 27], and anomaly detection.

The LeZi-update framework uses a symbolic space to represent sensing zone of the smart environment as an alphabetic symbol and thus captures inhabitant's movement history as a string of symbols. That is, while the geographic location data are often useful in obtaining precise location coordinates, the symbolic information removes the burden of frequent coordinate translation and is capable of achieving universality across different networks [24, 27]. The blessing of symbolic representation also helps us hierarchically abstract the indoor connectivity infrastructure into different levels of granularity. We assume that the inhabitants' itineraries are inherently compressible and allow application of universal data compression algorithms [31], which make very basic and broad assumptions, and yet minimize the source entropy for stationary Ergodic stochastic processes [26].

In LeZi-update, the symbols (sensor-ids) are processed in chunks and the entire sequence of symbols withheld until the last update is reported in a compressed (encoded) form. For example, referring to the abstract representation of mobility in Figure 2(a), let the inhabitant's movement history at any instant be given as ajlloojhhaajlloojaajlloojaajll... . This string of symbols can be parsed as distinct substrings (or phrases) “a, j, l, lo, o, jh, h, aa, jl, loo, ja, aj, 1l, oo, jaa, jll, ...”. As shown in 
Figure 2(b), such a symbol-wise context model, based on variable-to-fixed length coding, can be efficiently stored in a dictionary implemented by a trie. Essentially, the mobile node acts as an encoder while the system acts as a decoder and the frequency of every symbol is incremented for every prefix of every suffix of each phrase. By accumulating larger and larger contexts, one can affect a paradigm shift from traditional position update to route update. For stationary Ergodic sources with $n$ symbols, this framework achieves asymptotic optimality, with improved location update cost bounded by $o(\lg n-\lg \lg n)$ where $\lg n$ denotes $\operatorname{logarithm}$ base 2 .
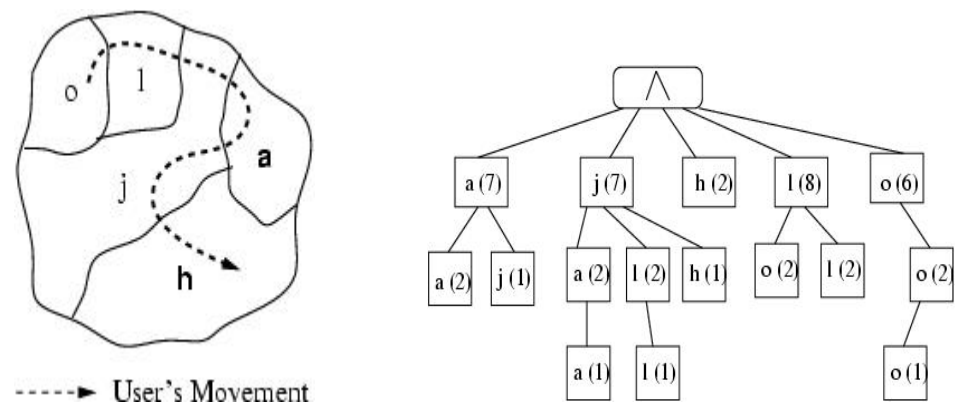

Fig. 2. (a) Symbolic representation of mobility, (b) Trie holding zones and their frequencies

Table 1. Phrases and their frequencies at context "jl", "j" and $\Lambda$

\begin{tabular}{lllll}
\hline $\mathrm{j} 1$ & $\mathrm{j}$ & $\Lambda$ & & \\
\hline $\operatorname{llj} 1(1)$ & $\mathrm{alj}(1)$ & $\mathrm{a}(4)$ & $\mathrm{aa}(2)$ & $\mathrm{aj}(1)$ \\
$\Lambda \mathrm{jj} 1(1)$ & $\mathrm{aalj}(1)$ & $\mathrm{j}(2)$ & $\mathrm{ja}(1)$ & $\mathrm{jaa}(1)$ \\
& $\operatorname{Llj}(1)$ & $\mathrm{jl}(1)$ & $\mathrm{jh}(1)$ & $1(4)$ \\
& $\operatorname{lllj}(1)$ & $\mathrm{lo}(1)$ & $\operatorname{loo}(1)$ & $\mathrm{ll}(2)$ \\
& $\mathrm{hlj}(1)$ & $\mathrm{o}(4)$ & $\mathrm{oo}(2)$ & $\mathrm{h}(2)$ \\
& $\Lambda \mathrm{lj}(2)$ & $\Lambda(1)$ & & \\
\hline
\end{tabular}

One major objective of the LeZi-update scheme is to endow the prediction process, by which the system finds nodes whose position is uncertain, with sufficient information regarding the node mobility profile. Each node in the trie preserves the relevant frequencies provided by the update mechanism in the current context. Thus, considering "jll" as the latest update phrase (route), the usable contexts are its prefixes: "jl", " $\mathrm{j}$ " and $\Lambda$ (null symbol). A list of all predictable contexts with frequencies is shown in Table 1. Following the blending technique of prediction by partial match (PPM) [6], the probability computation starts from the leaf nodes (highest level) of the trie and escapes to the lower levels until the root is reached. Based on the principle of insufficient reasoning [26], every phrase probability is distributed among individual symbols (zones) according to their relative occurrence in a particular phrase. The total residence probability of every zone (symbol) is computed by adding the accumulated 
probabilities from all possible phrases at this context. The optimal prediction order is now determined by polling the zones in decreasing order of residence probabilities.

So overall, the application of information-theoretic methods to location prediction allowed quantification of minimum information exchanges to maintain accurate location information, provided an on-line method by which to characterize mobility, and in addition, endowed an optimal prediction sequence [11]. Through learning this approach allows us to build a higher order mobility model rather than assuming a finite model, and thus minimizes entropy and leads to optimal performance.

While the basic LeZi-Update algorithm was used to predict only the current location from past movement patterns, this approach has also been extended in [28] to predict the likely future routes (or trajectories) of inhabitants in smart homes and also for heterogeneous environments [23]. The route prediction exploits the asymptotic equi-partition property in information theory [8], which implies the algorithm predicts a relatively small set (called typical set) of routes that the user is likely to take. A smart home environment can then act on this information by activating resources in an efficient manner (for example, by turning on the lights lying only on these routes). Our experiments [28] demonstrate that the predictive framework can save up to $70 \%$ electrical energy in a typical smart home environment. The prediction accuracy is up to $86 \%$ while only $11 \%$ of routes constitute the typical set.

\subsection{Inhabitant Action Prediction}

A smart home inhabitant typically interacts with various devices as part of routine activities. These interactions may be considered as a sequence of events, with some inherent repeatability pattern, that can be modeled as a stationary stochastic process. Inhabitant action prediction consists of first mining the data to identify sequences of actions that are regular and repeatable enough to generate predictions, and using a sequence matching approach to predict the next action.

To mine the data, a window can be moved in a single pass through the history of inhabitant actions, looking for sequences within the window that merit attention. Each sequence is evaluated using the Minimum Description Length principle [26], which favors sequences that minimize the description length of the sequence once it is compressed by replacing each instance of the discovered pattern with a pointer to the pattern definition. A regularity factor (daily, weekly, monthly) helps compress the data and thus increases the value of a pattern. Action sequences are first filtered by the mined sequences. If a sequence is considered significant by the mining algorithm, then predictions can be made for events within the sequence window. Using this algorithm as a filter for two alternative prediction algorithms, the resulting accuracy increases on an average by $50 \%$. This filter ensures that MavHome will not erroneously seek to automate anomalous and highly variable activities $[18,19]$.

As above, the action prediction algorithm parses the input string (history of interactions) into substrings representing phrases. Because of the prefix property used by the algorithm, parsed substrings can be efficiently maintained in a trie along with the frequency information. To perform prediction, the algorithm calculates the probability of each symbol (action) occurring in the parsed sequence, and predicts the action with the highest probability. To achieve optimal predictability, the predictor must use a mixture of all possible order models (phrase sizes) when determining the probability 
estimate. To accomplish this, techniques from the PPM family of predictors are incorporated, that generate weighted Markov models of different orders. This blending strategy assigns greater weight to higher-order models, in keeping with the advisability of making the most informed decision.

In our experiments run on sample smart home data, predictive accuracy of this approach converged on $100 \%$ for perfectly-repeatable data with no variation, and converged on $86 \%$ accuracy for data containing variations and anomalies [16].

\subsection{Automated Decision Making}

The goal of MavHome is to enable automation of basic functions so as to maximize the inhabitants' comfort and minimize the operating cost of the home. We assume comfort is a function of the number of manual interactions with the home, and the operating cost of energy usage. Because the goal is a combination of these two factors, blind automation of all inhabitant actions is frequently not the desired solution. For example, an inhabitant might turn on the hallway light in the morning before opening the blinds in the living room. MavHome could, on the other hand, open the blinds in the living room before the inhabitant leaves the bedroom, thus alleviating the need for the hallway lights. Similarly, turning down the air conditioning after leaving the house and turning it back up before returning would be more energy efficient than turning the air conditioning to maximum after arriving home in order to cool it as quickly as possible [28].

To achieve its goal, MavHome uses reinforcement learning to acquire an optimal decision policy. In this framework, the agent learns autonomously from potentially delayed rewards rather than from a teacher, reducing the requirement for the home's inhabitant to supervise or program the system. To learn a strategy, the agent explores the effects of its actions over time and uses this experience to form control policies that optimize the expected future reward.

\section{Conclusion}

This paper summarizes our experience on the effectiveness of learning and prediction based paradigm in designing a smart home environment. Efficient prediction algorithms provide information useful for future locations and activities, automating activities, optimizing design and control methods for devices and tasks within the environment, and identifying anomalies. These technologies reduce the work to maintain a home, lessen energy utilization, and provide special benefits for elderly and people with disabilities. In the future, these abilities will be generalized to conglomeration of environments, including smart offices, smart roads, smart hospitals, smart automobiles, and smart airports, through which a user may pass through in daily life. Another research challenge is how to characterize mobility and activity profiles of multiple inhabitants (e.g., living in the same home) in the same dictionary and predict or trigger actions to meet the common goals of the house under conflicting requirements of individual inhabitants.

Acknowledgements. This research was supported by the US National Science Foundation grants under award numbers IIS-0121297 and IIS-0326505. 


\section{References}

[1] G. D. Abowd, "Classroom 2000: An Experiment with the Instrumentation of a Living educational Environment,” IBM Systems Journal, 38(4), pp, 508-530, 1999.

[2] A. Bhattacharya and S. K. Das, "LeZi-Update: An Information-Theoretic Approach for Personal Mobility Tracking in PCS Networks," Wireless Networks, 8, pp. 121-135, 2002.

[3] A. Bobick, et al., "The KidsRoom: A Perceptually-Based Interactive and Immersive Story Environment," Presence, 8 (4), pp. 369-393, Aug 1999.

[4] B. Brumitt, et al., "Ubiquitous Computing and the Role of Geometry," IEEE Personal Communications, 7 (5), pp. 41-43, Aug 2000.

[5] Cisco, http://www.cisco.com/warp/public//3/uk/ihome

[6] J. G. Cleary and I. H. Witten, "Data Compression Using Adaptive Coding and Partial String Matching," IEEE Transactions on Communications, 32 (4), pp. 396-402, Apr 1984.

[7] D. J. Cook and S. K. Das, Smart Environments: Technology, Protocols, and Applications, Wiley, 2005.

[8] T. M. Cover and J. A. Thomas, Elements of Information Theory, Wiley, 1991.

[9] S. K. Das, D. J. Cook, et al., "The Role of Prediction Algorithms in the MavHome Smart Home Architecture," IEEE Wireless Communications, 9 (6), pp. 77-84, Dec 2002.

[10] S. K. Das and D. J. Cook, “Agent Based Health Monitoring in Smart Homes," Proc Int. Conf. on Smart Homes and Health Telematics (ICOST), Singapore, Sept 2004 (Keynote Talk).

[11] S. K. Das and C. Rose, "Coping with Uncertainty in Wireless Mobile Networks," Proc. of IEEE Personal, Indoor and Mobile Radio Communications, Barcelona, Spain, Sept 2004 (Invited Talk).

[12] Edinvar, http://www.stakes.fi/tidecong/732bonne.html

[13] A. Fox, B. Johanson, P. Hanrahan, T. Winograd, "Integrating Information Appliances into an Interactive Space," IEEE Computer Graphics and Applications, 20 (3), pp. 54-65, 2000.

[14] http://www.dementua-voice.org.uk/Projects_GloucesterProject.html

[15] K. Gopalratnam and D. J. Cook, "Online Sequential Prediction via Incremental Parsing: The Active LeZi Algorithm”, IEEE Intelligent Systems, 2005.

[16] K. Gopalratnam and D. J. Cook, "Active LeZi: An Incremental Parsing Algorithm for Sequential Prediction", International Journal of Artificial Intelligence Tools, 14 (1-2), 2004.

[17] S. Helal, et al., "Enabling Location-Aware Pervasive Computing Applications for the Elderly," Proc. of IEEE Int. Conf. on Pervasive Computing and Communications (PerCom'03), pp. 531-538, Mar 2003.

[18] E. Heierman, M. Youngblood, and D. J. Cook, "Mining Temporal Sequences to Discover Interesting Patterns", KDD Workshop on Mining Temporal and Sequential Data, 2004.

[19] E. Heierman and D. J. Cook, "Improving Home Automation by Discovering Regularly Occurring Device Usage Patterns", Proc. of International Conf. on Data Mining, 2003.

[20] Intel, http://www.intel.com/research/prohealth

[21] C. Kidd, et al., "The Aware Home: A Living Laboratory for Ubiquitous Computing," Proceedings of the Second International Workshop on Cooperative Buildings, 1999.

[22] C. Le Gal, J. Martin, A. Lux and J. L. Crowley, "Smart Office: Design of an Intelligent Environment," IEEE Intelligent Systems, 16 (4), July-Aug 2001.

[23] A. Misra, A. Roy and S. K. Das, "An Information Theoretic Framework for Optimal Location Tracking in Multi-System 4G Wireless Networks," Proc. IEEE INFOCOM, 2004. 
[24] A. Misra and S. K. Das, "Location Estimation (Determination and Prediction) Techniques in Smart Environments," Smart Environments (Eds. D. J. Cook and S. K. Das), Ch. 8, pp. 193-228, Wiley, 2005.

[25] M. Mozer, "The Neural Network House: An Environment that Adapts to its Inhabitants," Proc. of the AAAI Spring Symposium on Intelligent Environments, 1998.

[26] J. Rissanen, Stochastic Complexity in Statistical Inquiry, World Scientific, 1989.

[27] A. Roy, S. K. Das and A. Misra, "Exploiting Information Theory for Adaptive Mobility and Resource Management in Future Wireless Cellular Networks," IEEE Wireless Commun., 11 (8), pp. 59-64, 2004.

[28] A. Roy, S. K. Das, et al., "Location Aware Resource Management in Smart Homes," Proc. IEEE Int. Conf. on Pervasive Computing and Communications, pp. 481-488, 2003.

[29] Smart sofa, http://www.dsg.cs.tcd.ie/?category_id=350

[30] M. B. Srivastava, et al., "Smart Kindergarten: Sensor-Based Wireless Networks for Smart Problem-Solving Environments," Proc. ACM Int. Conf. on Mobile Computing and Networking, Rome, July 2001.

[31] J. Ziv and A. Lempel, "Compression of Individual Sequences via Variable Rate Coding," IEEE Transcations on Information Theory, 24(5), pp. 530-536, Sept 1978.

[32] M. Youngblood, D. J. Cook, and L. B. Holder, "Managing Adaptive Versatile Environments", Proc. IEEE Int. Conf. on Pervasive Computing and Communications, 2005. 